\title{
COMPARISON THEOREMS IN FINSLER GEOMETRY WITH WEIGHTED CURVATURE BOUNDS AND RELATED RESULTS
}

\author{
BING-YE Wu
}

\begin{abstract}
We first extend the notions of weighted curvatures, including the weighted flag curvature and the weighted Ricci curvature, for a Finsler manifold with given volume form. Then we establish some basic comparison theorems for Finsler manifolds with various weighted curvature bounds. As applications, we obtain some McKean type theorems for the first eigenvalue of Finsler manifolds, some results on weighted curvature and fundamental group for Finsler manifolds, as well as an estimation of Gromov simplicial norms for reversible Finsler manifolds.
\end{abstract}

\section{Introduction}

Comparison technique is a powerful tool in global analysis in differential geometry, and it has been well developed in Riemannian geometry. Among these issues, the Laplacian comparison theorems and volume comparison theorems are important and interesting, and one can derive the volume comparison results from Laplacian comparison theorems. Recently comparison technique has been developed for Finsler manifolds and the relationship between curvature and topology of Finsler manifolds has also been investigated [2, 12, 13, 14, 17]. As in the Riemannian case, the Laplacian of a smooth function on a Finsler manifold is defined as the divergence of the gradient of the function, and it depends on the choice of the volume form. Since there are different choices of volume forms for given Finsler metrics, we usually need to control the Scurvature in order to obtain Laplacian comparison theorems as well as the volume comparison theorems.

It should be noted here that by utilizing the weighted Ricci curvature condition, Ohta and Sturm $[9,10]$ gave another version of Laplacian comparison theorem and volume comparison theorem, which absorb the S-curvature into the weighted Ricci curvature assumption and remove the S-curvature in the

Received August 26, 2014.

2010 Mathematics Subject Classification. Primary 53C60; Secondary 53B40.

Key words and phrases. Finsler manifold, weighted flag curvature, weighted Ricci curvature, first eigenvalue, fundamental group. 
conclusion. In this paper we shall continue investigations in this direction. We first extend the notions of weighted curvatures, including the weighted flag curvature and the weighted Ricci curvature, for a Finsler manifold with given volume form. Our notions include Ohta's notion of weighted Ricci curvature as a special case. Then we shall establish some weighted Hessian comparison theorems, Laplacian comparison theorems and volume comparison theorems for Finsler manifolds under various weighted curvature assumptions. As their applications, we obtain some McKean type theorems for the first eigenvalue of Finsler manifolds, some results on weighted curvature and fundamental group for Finsler manifolds, as well as an estimation of Gromov simplicial norms for reversible Finsler manifolds. Our results indicate that it is quite natural to introduce the notions of weighted curvatures from viewpoint of comparison geometry.

\section{Finsler geometry}

Let $(M, F)$ be a Finsler $n$-manifold with Finsler metric $F: T M \rightarrow[0, \infty)$. Let $(x, y)=\left(x^{i}, y^{i}\right)$ be local coordinates on $T M$, and $\pi: T M \backslash 0 \rightarrow M$ the natural projection. Unlike in the Riemannian case, most Finsler quantities are functions of $T M$ rather than $M$. The fundamental tensor $g_{i j}$ and the Cartan tensor $C_{i j k}$ are defined by

$$
g_{i j}(x, y):=\frac{1}{2} \frac{\partial^{2} F^{2}(x, y)}{\partial y^{i} \partial y^{j}}, \quad C_{i j k}(x, y):=\frac{1}{4} \frac{\partial^{3} F^{2}(x, y)}{\partial y^{i} \partial y^{j} \partial y^{k}} .
$$

Let $\Gamma_{j k}^{i}(x, y)$ be the Chern connection coefficients. Then the first Chern curvature tensor $R_{j k l}{ }^{i}$ can be expressed by

$$
R_{j k l}^{i}=\frac{\delta \Gamma_{j l}^{i}}{\delta x^{k}}-\frac{\delta \Gamma_{j k}^{i}}{\delta x^{l}}+\Gamma_{k s}^{i} \Gamma_{j l}^{s}-\Gamma_{j k}^{s} \Gamma_{l s}^{i},
$$

where $\frac{\delta}{\delta x^{i}}:=\frac{\partial}{\partial x^{i}}-y^{k} \Gamma_{i k}^{j} \frac{\partial}{\partial y^{j}}$. Let $R_{i j k l}:=g_{j s} R_{i k l}{ }^{s}$, and write $\mathbf{g}_{y}=g_{i j}(x, y) d x^{i} \otimes$ $d x^{j}, \mathbf{R}_{y}=R_{i j k l}(x, y) d x^{i} \otimes d x^{j} \otimes d x^{k} \otimes d x^{l}$. The angular metric form $\mathbf{h}_{y}$ is defined by

$$
\mathbf{h}_{y}(u, v)=\mathbf{g}_{y}(u, v)-\frac{1}{F^{2}(y)} \mathbf{g}_{y}(y, u) \mathbf{g}_{y}(y, v), \quad \forall y, u, v \in T_{x} M \text { with } y \neq 0 .
$$

For a tangent plane $P \subset T_{x} M$, let

$$
\mathbf{K}(P, y)=\mathbf{K}(y ; u):=\frac{\mathbf{R}_{y}(y, u, u, y)}{F^{2}(y) \mathbf{h}_{y}(u, u)}=\frac{\mathbf{R}_{y}(y, u, u, y)}{\mathbf{g}_{y}(y, y) \mathbf{g}_{y}(u, u)-\left[\mathbf{g}_{y}(y, u)\right]^{2}},
$$

where $y, u \in P$ are tangent vectors such that $P=\operatorname{span}\{y, u\} . \mathbf{K}(P, y)$ is called the flag curvature of $P$ with flag pole $y$. Let

$$
\boldsymbol{R i c}(y)=\sum_{i=1}^{n-1} \mathbf{K}\left(y ; e_{i}\right)
$$


here $e_{1}, \ldots, e_{n-1}, e_{n}=y$ is a $\mathbf{g}_{y}$-orthogonormal basis for the corresponding tangent space. $\operatorname{Ric}(y)$ is called the Ricci curvature of $y$.

Let $V=v^{i} \partial / \partial x^{i}$ be a non-vanishing vector field on an open subset $\mathcal{U} \subset M$. One can introduce a Riemannian metric $\widetilde{g}=\mathbf{g}_{V}$ and a linear connection $\nabla^{V}$ (called Chern connection) on the tangent bundle over $\mathcal{U}$ as follows:

$$
\nabla_{\frac{\partial}{\partial x^{i}}}^{V} \frac{\partial}{\partial x^{j}}:=\Gamma_{i j}^{k}(x, v) \frac{\partial}{\partial x^{k}} .
$$

From the torsion freeness and almost g-compatibility of Chern connection we have

$$
\begin{gathered}
\nabla_{X}^{V} Y-\nabla_{Y}^{V} X=[X, Y] \\
X \cdot \mathbf{g}_{V}(Y, Z)=\mathbf{g}_{V}\left(\nabla_{X}^{V} Y, Z\right)+\mathbf{g}_{V}\left(Y, \nabla_{X}^{V} Z\right)+2 \mathbf{C}_{V}\left(\nabla_{X}^{V} V, Y, Z\right),
\end{gathered}
$$

here $\mathbf{C}_{V}=C_{i j k}(x, v) d x^{i} \otimes d x^{j} \otimes d x^{k}$, and it satisfies

$$
\mathbf{C}_{V}(V, X, Y)=0 \text {. }
$$

Given a Finsler manifold $(M, F)$, the dual Finsler metric $F^{*}$ on $M$ is defined by

$$
F^{*}\left(\xi_{x}\right):=\sup _{Y \in T_{x} M \backslash 0} \frac{\xi(Y)}{F(Y)}, \quad \forall \xi \in T^{*} M,
$$

and the corresponding fundamental tensor is defined by

$$
g^{* k l}(\xi)=\frac{1}{2} \frac{\partial^{2} F^{* 2}(\xi)}{\partial \xi_{k} \partial \xi_{l}} .
$$

The Legendre transformation $l: T M \rightarrow T^{*} M$ is defined by

$$
l(Y)= \begin{cases}\mathbf{g}_{Y}(Y, \cdot), & Y \neq 0 \\ 0, & Y=0\end{cases}
$$

It is well-known that for any $x \in M$, the Legendre transformation is a smooth diffeomorphism from $T_{x} M \backslash 0$ onto $T_{x}^{*} M \backslash 0$, and it is norm-preserving, namely, $F(Y)=F^{*}(l(Y)), \forall Y \in T M$. Consequently, $g^{i j}(Y)=g^{* i j}(l(Y))$.

Now let $f: M \rightarrow \mathbb{R}$ be a smooth function on $M$. The gradient of $f$ is defined by $\nabla f=l^{-1}(d f)$. Thus we have

$$
d f(X)=\mathbf{g}_{\nabla f}(\nabla f, X), \quad X \in T M .
$$

Let $\mathcal{U}=\left\{x \in M:\left.\nabla f\right|_{x} \neq 0\right\}$. We define the Hessian $H(f)$ of $f$ on $\mathcal{U}$ as follows:

$$
H(f)(X, Y):=X Y(f)-\nabla_{X}^{\nabla f} Y(f), \quad \forall X,\left.Y \in T M\right|_{\mathcal{U}}
$$

It is known that $H(f)$ is symmetric, and it can be rewritten as (see [17])

$$
H(f)(X, Y)=\mathbf{g}_{\nabla f}\left(\nabla_{X}^{\nabla f} \nabla f, Y\right)
$$

It should be noted that the notion of Hessian defined here is different from that in [13]. In that case $H(f)$ is in fact defined by

$$
H(f)(X, X)=X \cdot X \cdot(f)-\nabla_{X}^{X} X(f),
$$


and there is no definition for $H(f)(X, Y)$ if $X \neq Y$. The advantage of our definition is that $H(f)$ is a symmetric bilinear form and we can treat it by using the theory of symmetric matrix.

Let $\gamma(t), 0 \leq t \leq l$ be a geodesic with unit speed velocity field $T$. A vector field $J$ along $\gamma$ is called a Jacobi field if it satisfies the following equation

$$
\nabla_{T}^{T} \nabla_{T}^{T} J+\mathbf{R}^{T}(J, T) T=0 .
$$

$J$ is called normal if $\mathbf{g}_{T}(T, J)=0$. For vector fields $X$ and $Y$ along $\gamma$, the index form $I_{\gamma}(X, Y)$ is defined by

$$
I_{\gamma}(X, Y)=\int_{0}^{l}\left(\mathbf{g}_{T}\left(\nabla_{T}^{T} X, \nabla_{T}^{T} Y\right)-\mathbf{g}_{T}\left(\mathbf{R}^{T}(X, T) T, Y\right)\right) d t .
$$

Let $r=d_{F}(p, \cdot)$ be the distance function from $p \in M$. Suppose that $r$ is smooth at $q \in M$, and $X \in T_{q} M$. Then we have

$$
H(r)(X, X)=I_{\gamma}(J, J),
$$

here $\gamma$ is the minimal geodesic from $p$ to $q$, and $J$ is the unique Jacobi field determined by $J(0)=0, J(r(q))=X$ (see e.g., (4.1) of [17]).

A volume form $d \mu$ on Finsler manifold $(M, F)$ is nothing but a global nondegenerate $n$-form on $M$. In local coordinates we can express $d \mu$ as $d \mu=$ $\sigma(x) d x^{1} \wedge \cdots \wedge d x^{n}$. For $y \in T_{x} M \backslash 0$, define

$$
\tau(y):=\log \frac{\sqrt{\operatorname{det}\left(g_{i j}(x, y)\right)}}{\sigma(x)} .
$$

$\tau$ is called the distortion of $(M, F, d \mu)$. To measure the rate of distortion along geodesics, we define

$$
\mathbf{S}(y):=\dot{\tau}(y):=\frac{d}{d t}[\tau(\dot{\gamma}(t))]_{t=0},
$$

where $\gamma(t)$ is the geodesic with $\dot{\gamma}(0)=y$. $\mathbf{S}$ is called the $S$-curvature $[12,13]$, and it is an important measure of the non-Riemannian curvature for Finsler manifolds. In what follows we consider the Finsler manifold $(M, F, d \mu)$ equipped with a volume form $d \mu$. Let $X \in T M$. The divergence $\operatorname{div}(X)$ of $X$ is defined as

$$
d(X\rfloor d \mu)=\operatorname{div}(X) d \mu .
$$

For a given smooth function $f: M \rightarrow \mathbb{R}$, the Laplacian $\Delta f$ of $f$ is defined by $\Delta f=\operatorname{div}(\nabla f)=\operatorname{div}\left(l^{-1}(d f)\right)$. We have:

Lemma 2.1 ([17]). Let $(M, F, d \mu)$ be a Finsler n-manifold, and $f: M \rightarrow \mathbb{R} a$ smooth function on $M$. Then on $\mathcal{U}=\left\{x \in M:\left.\nabla f\right|_{x} \neq 0\right\}$ we have

$$
\Delta f=\sum_{a} H(f)\left(e_{a}, e_{a}\right)-\mathbf{S}(\nabla f):=\operatorname{tr}_{\nabla f} H(f)-\mathbf{S}(\nabla f),
$$

where $e_{1}, \ldots, e_{n}$ is a local $\mathbf{g}_{\nabla f}$-orthonormal frame on $\mathcal{U}$. 


\section{Weighted Hessian comparison theorems}

Motivated by Lemma 2.1 we define the weighted Hessian $\widetilde{H}(f)$ of a smooth $f$ on $\mathcal{U}=\left\{x \in M:\left.\nabla f\right|_{x} \neq 0\right\}$ by

$$
\widetilde{H}(f)(X, Y)=H(f)(X, Y)-\frac{\mathbf{S}(\nabla f)}{n-1} \mathbf{h}_{\nabla f}(X, Y), \quad \forall X,\left.Y \in T M\right|_{\mathcal{U}}
$$

then it is clear that $\widetilde{H}(f)$ is still a symmetric bilinear form with

$$
\Delta f=\operatorname{tr}_{\nabla f} \widetilde{H}(f) .
$$

In the following we shall consider the weighted Hessian of distance function $r=d_{F}(p, \cdot)$ from a fixed point $p \in M$. We note that the gradient $\nabla r$ is a unit geodesic field where $r$ is smooth.

Lemma 3.1. Let $r=d_{F}(p, \cdot)$ be smooth at $q \in M$, and $X, Y \in T_{q} M$. Then

$$
\widetilde{H}(r)(X, Y)=\widetilde{H}(r)\left(X^{\perp}, Y^{\perp}\right),
$$

where $X^{\perp}:=X-\mathbf{g}_{\nabla r}(\nabla r, X) \nabla r$.

Proof. By the definition of angular metric form it is clear that $\mathbf{h}_{\nabla r}(X, Y)=$ $\mathbf{h}_{\nabla r}\left(X^{\perp}, Y^{\perp}\right)$, thus we need only to prove $H(r)(X, Y)=H(r)\left(X^{\perp}, Y^{\perp}\right)$. Since $H(r)$ is a symmetric bilinear form, we have

$$
\begin{aligned}
H(r)(X, Y)= & H(r)\left(X^{\perp}+\mathbf{g}_{\nabla r}(\nabla r, X) \nabla r, Y^{\perp}+\mathbf{g}_{\nabla r}(\nabla r, Y) \nabla r\right) \\
= & H(r)\left(X^{\perp}, Y^{\perp}\right)+\mathbf{g}_{\nabla r}(\nabla r, X) H(r)\left(\nabla r, Y^{\perp}\right) \\
& +\mathbf{g}_{\nabla r}(\nabla r, Y) H(r)\left(\nabla r, X^{\perp}\right) \\
& +\mathbf{g}_{\nabla r}(\nabla r, X) \mathbf{g}_{\nabla r}(\nabla r, Y) H(r)(\nabla r, \nabla r) .
\end{aligned}
$$

Recall that $\nabla r$ is a geodesic field, by (2.4) one has

$$
H(r)(\nabla r, \cdot)=\mathbf{g}_{\nabla r}(\nabla \nabla r \nabla r, \cdot)=0,
$$

and so we are done.

By Lemma 3.1 we need only to consider $\widetilde{H}(r)$ on the normal space with respect to the radial geodesic field $\nabla r$. Now we are ready to prove the weighted Hessian comparison theorem as follows.

Theorem 3.2. Let $(M, F, d \mu)$ be a forward complete Finsler $n$-manifold, $r=$ $d_{F}(p, \cdot)$, the distance function from a fixed point $p$. Suppose that for some $1<N<n$, the flag curvature $\mathbf{K}$ and $S$-curvature $\mathbf{S}$ of $M$ satisfy

$\mathbf{K}(V ; W)+\frac{\dot{\mathbf{S}}(V)}{(n-1) F^{2}(V)}-\frac{(\mathbf{S}(V))^{2}}{(n-1)(N-n) F^{2}(V)} \leqslant \frac{N-1}{n-1} \cdot c, \quad \forall V, W \in T M$,

where $\dot{\mathbf{S}}$ is the geodesic differentiation of $\mathbf{S}$. Then, for any vector field $X$ on $M$, the following inequality holds whenever $r$ is smooth $(r<\pi / \sqrt{c}$ when $c>0)$ :

$$
\widetilde{H}(r)(X, X) \geqslant \frac{N-1}{n-1} \cdot \mathfrak{c t}_{c}(r)\left(\mathbf{g}_{\nabla r}(X, X)-\mathbf{g}_{\nabla r}(\nabla r, X)^{2}\right),
$$


where

$$
\mathfrak{c t}_{c}(r)= \begin{cases}\sqrt{c} \cdot \cot \sqrt{c} r, & c>0 \\ \frac{1}{r}, & c=0 \\ \sqrt{-c} \cdot \operatorname{coth} \sqrt{-c} r, & c<0\end{cases}
$$

Proof. Suppose that $r$ is smooth at $q \in M$, and $\gamma:[0, l] \rightarrow M$ is the unique minimal geodesic from $p$ to $q$, where $l=r(q)$. Let $J=J(r), 0 \leqslant r \leqslant l$ be the unique normal Jacobi field along $\gamma$ with $J(0)=0$ and $J(l)=X^{\perp}(q)$. Then by Lemma 3.1, (2.5) and (3.1) we have

$$
\begin{aligned}
& \widetilde{H}(r)(X(q), X(q)) \\
= & -\frac{\left.\mathbf{S}(\nabla r)\right|_{q}}{n-1} \mathbf{g}_{\nabla r}\left(X^{\perp}(q), X^{\perp}(q)\right) \\
& +\int_{0}^{l}\left[\mathbf{g}_{\nabla r}\left(\nabla \nabla_{\nabla r}^{\nabla r} J, \nabla_{\nabla r}^{\nabla r} J\right)-\mathbf{g}_{\nabla r}(J, J) \mathbf{K}(\nabla r ; J)\right] d r .
\end{aligned}
$$

On the other hand, integrating by parts we get

$$
\begin{aligned}
& \int_{0}^{l} \mathbf{g}_{\nabla r}(J, J) \cdot \dot{\mathbf{S}}(\nabla r) d r \\
= & {\left.\left[\mathbf{S}(\nabla r) \mathbf{g}_{\nabla r}(J, J)\right]\right|_{0} ^{l}-\int_{0}^{l} 2 \mathbf{g}_{\nabla r}\left(\nabla \nabla_{\nabla r}^{\nabla r} J, J\right) \mathbf{S}(\nabla r) d r } \\
= & \left.\mathbf{S}(\nabla r)\right|_{q} \cdot \mathbf{g}_{\nabla r}\left(X^{\perp}(q), X^{\perp}(q)\right)-\int_{0}^{l} 2 \mathbf{g}_{\nabla r}(\nabla \nabla r r, J) \mathbf{S}(\nabla r) d r,
\end{aligned}
$$

which together with (3.3) yields

$$
\begin{aligned}
& \widetilde{H}(r)(X(q), X(q)) \\
= & \int_{0}^{l}\left[\mathbf{g}_{\nabla r}\left(\nabla \frac{\nabla r}{\nabla r} J, \nabla \frac{\nabla r}{\nabla r} J\right)-\mathbf{g}_{\nabla r}(J, J)\left(\mathbf{K}(\nabla r ; J)+\frac{\dot{\mathbf{S}}(\nabla r)}{n-1}\right)\right. \\
& \left.-\frac{2}{n-1} \mathbf{g}_{\nabla r}(\nabla \nabla \nabla r J, J) \mathbf{S}(\nabla r)\right] d r .
\end{aligned}
$$

Plugging

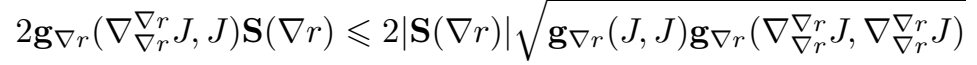

$$
\begin{aligned}
& \leqslant \frac{(\mathbf{S}(\nabla r))^{2}}{n-N} \cdot \mathbf{g}_{\nabla r}(J, J)+(n-N) \cdot \mathbf{g}_{\nabla r}\left(\nabla_{\nabla r}^{\nabla r} J, \nabla \underset{\nabla r}{\nabla r} J\right)
\end{aligned}
$$

into (3.4) we have

$$
\begin{aligned}
& \widetilde{H}(r)(X(q), X(q)) \\
\geqslant & \int_{0}^{l}\left[\frac{N-1}{n-1} \mathbf{g}_{\nabla r}\left(\nabla_{\nabla r}^{\nabla r} J, \nabla_{\nabla r}^{\nabla r} J\right)\right.
\end{aligned}
$$




$$
\begin{aligned}
& \left.-\mathbf{g}_{\nabla r}(J, J)\left(\mathbf{K}(\nabla r ; J)+\frac{\dot{\mathbf{S}}(\nabla r)}{n-1}-\frac{(\mathbf{S}(\nabla r))^{2}}{(n-1)(N-n)}\right)\right] d r \\
& \geqslant \frac{N-1}{n-1} \int_{0}^{l}\left[\mathbf{g}_{\nabla r}(\nabla \nabla r r)\right.
\end{aligned}
$$

Let $E_{1}(r), \ldots, E_{n-1}(r), E_{n}(r)=\nabla r$ be $\mathbf{g}_{\nabla r}$-orthonormal frame that is parallel along $\gamma$, and $E_{1}(l)=\frac{J(l)}{\sqrt{\mathbf{g} \nabla r(J(l), J(l))}}$. Write

$$
J(r)=J^{1}(r) E_{1}(r)+\cdots+J^{n-1}(r) E_{n-1}(r), \quad 0 \leqslant r \leqslant l .
$$

Let $(\bar{M}, \bar{g})$ be a Riemannian space form of constant $c$, and $\bar{\nabla}$ be the corresponding Levi-Civita connection. Fix a normal geodesic $\bar{\gamma}:[0, l] \rightarrow \bar{M}$, and construct a vector field $\bar{Y}$ along $\bar{\gamma}$ by

$$
\bar{Y}(r)=J^{1}(r) \bar{E}_{1}(r)+\cdots+J^{n-1}(r) \bar{E}_{n-1}(r), \quad 0 \leqslant r \leqslant l,
$$

where $\bar{E}_{1}(r), \ldots, \bar{E}_{n-1}(r), \bar{E}_{n}(r)=\dot{\bar{\gamma}}$ be the $\bar{g}$-orthonormal frame that is parallel along $\bar{\gamma}$. By construction we clearly have, for any $0 \leqslant r \leqslant l$,

$$
\begin{gathered}
\mathbf{g}_{\nabla r}(J, J)(r)=\left(J^{1}(r)\right)^{2}+\cdots+\left(J^{n-1}(r)\right)^{2}=\bar{g}(\bar{Y}, \bar{Y})(r), \\
\mathbf{g}_{\nabla r}\left(\nabla_{\nabla r}^{\nabla r} J, \nabla_{\nabla r}^{\nabla r} J\right)(r)=\left(\dot{J}^{1}(r)\right)^{2}+\cdots+\left(\dot{J}^{n-1}(r)\right)^{2}=\bar{g}\left(\bar{\nabla} \dot{\bar{\gamma}} \bar{Y}, \bar{\nabla}_{\dot{\bar{\gamma}}} \bar{Y}\right)(r),
\end{gathered}
$$

thus by (3.5) we get

$$
\begin{aligned}
\widetilde{H}(r)(X(q), X(q)) & \geqslant \frac{N-1}{n-1} \int_{0}^{l}\left[\bar{g}\left(\bar{\nabla} \dot{\bar{\gamma}} \bar{Y}, \bar{\nabla}_{\dot{\gamma}} \bar{Y}\right)-c \cdot \bar{g}(\bar{Y}, \bar{Y})\right] d r \\
& =\frac{N-1}{n-1} I_{\bar{\gamma}}(\bar{Y}, \bar{Y}),
\end{aligned}
$$

where $I_{\bar{\gamma}}$ is the index form on $\bar{\gamma}$. Since $(\bar{M}, \bar{g})$ has constant curvature $c$, the Jacobi field $\bar{J}$ on $\bar{\gamma}$ with $\bar{J}(0)=0=\bar{Y}(0)$ and $\bar{J}(l)=\bar{Y}(l)=\sqrt{\mathbf{g}_{\nabla r}(J(l), J(l))}$. $\bar{E}_{1}(l)$ is given by

$$
\bar{J}(r)=\frac{\mathfrak{s}_{c}(r)}{\mathfrak{s}_{c}(l)} \cdot \sqrt{\mathbf{g}_{\nabla r}(J(l), J(l))} \cdot \bar{E}_{1}(r)
$$

where

$$
\mathfrak{s}_{c}(t)= \begin{cases}\frac{1}{\sqrt{c}} \sin \sqrt{c} t, & c>0 \\ t, & c=0 \\ \frac{1}{\sqrt{-c}} \sinh \sqrt{-c} t, & c<0 .\end{cases}
$$

Now by Index Lemma (see e.g., [2]) and (3.6) we finally obtain

$$
\begin{aligned}
\widetilde{H}(r)(X(q), X(q)) & \geqslant \frac{N-1}{n-1} \cdot I_{\bar{\gamma}}(\bar{Y}, \bar{Y}) \geqslant \frac{N-1}{n-1} \cdot I_{\bar{\gamma}}(\bar{J}, \bar{J}) \\
& =\frac{N-1}{n-1} \mathfrak{c t}_{c}(l) \bar{g}(\bar{J}(l), \bar{J}(l))=\frac{N-1}{n-1} \mathfrak{c t}_{c}(l) \mathbf{g}_{\nabla r}(J(l), J(l)) \\
& =\frac{N-1}{n-1} \mathfrak{c t}_{c}(l) \mathbf{g}_{\nabla r}\left(X^{\perp}(q), X^{\perp}(q)\right)
\end{aligned}
$$




$$
=\frac{N-1}{n-1} \mathfrak{c t}_{c}(r(q))\left(\mathbf{g}_{\nabla r}(X(q), X(q))-\mathbf{g}_{\nabla r}(\nabla r, X(q))^{2}\right) .
$$

Theorem 3.3. Let $(M, F, d \mu)$ be a forward complete Finsler $n$-manifold, $r=$ $d_{F}(p, \cdot)$, the distance function from a fixed point $p$. Suppose that for some $N>n$, the flag curvature $\mathbf{K}$ and S-curvature $\mathbf{S}$ of $M$ satisfy

$\mathbf{K}(V ; W)+\frac{\dot{\mathbf{S}}(V)}{(n-1) F^{2}(V)}-\frac{(\mathbf{S}(V))^{2}}{(n-1)(N-n) F^{2}(V)} \geqslant \frac{N-1}{n-1} \cdot c, \quad \forall V, W \in T M$.

Then, for any vector field $X$ on $M$, the following inequality holds whenever $r$ is smooth:

$$
\widetilde{H}(r)(X, X) \leqslant \frac{N-1}{n-1} \cdot \mathfrak{c t}_{c}(r)\left(\mathbf{g}_{\nabla r}(X, X)-\mathbf{g}_{\nabla r}(\nabla r, X)^{2}\right) .
$$

Proof. Suppose that $r$ is smooth at $q \in M$, and let $\gamma, J$ be as in the proof of Theorem 3.2. Let $Y=Y(r)$ be a parallel vector field along $\gamma$ such that $Y(l)=X^{\perp}(q)$, and construct a vector field $W$ along $\gamma$ by

$$
W=W(r)=\frac{\mathfrak{s}_{c}(r)}{\mathfrak{s}_{c}(l)} \cdot Y(r),
$$

then $W(0)=0=J(0), W(l)=Y(l)=J(l)$. By Index Lemma, Lemma 3.1, (2.5) and (3.1) we have

$$
\begin{aligned}
\widetilde{H}(r)(X(q), X(q))= & -\frac{\left.\mathbf{S}(\nabla r)\right|_{q}}{n-1} \mathbf{g}_{\nabla r}\left(X^{\perp}(q), X^{\perp}(q)\right)+I_{\gamma}(J, J) \\
\leqslant & -\frac{\left.\mathbf{S}(\nabla r)\right|_{q}}{n-1} \mathbf{g}_{\nabla r}(W(l), W(l))+I_{\gamma}(W, W) \\
= & \mathbf{g}_{\nabla r}\left(X^{\perp}(q), X^{\perp}(q)\right)\left[-\frac{\left.\mathbf{S}(\nabla r)\right|_{q}}{n-1}\right. \\
& \left.\quad+\frac{1}{\left(\mathfrak{s}_{c}(l)\right)^{2}} \int_{0}^{l}\left(\left(\mathfrak{s}_{c}^{\prime}(r)\right)^{2}-\left(\mathfrak{s}_{c}(r)\right)^{2} \cdot \mathbf{K}(\nabla r ; W)\right) d r\right] .
\end{aligned}
$$

By the similar argument as in the proof of Theorem 3.2 we see that

$$
\begin{aligned}
-\frac{\left.\mathbf{S}(\nabla r)\right|_{q}}{n-1}+ & \frac{1}{\left(\mathfrak{s}_{c}(l)\right)^{2}} \int_{0}^{l}\left(\left(\mathfrak{s}_{c}^{\prime}(r)\right)^{2}-\left(\mathfrak{s}_{c}(r)\right)^{2} \cdot \mathbf{K}(\nabla r ; W)\right) d r \\
=\frac{1}{\left(\mathfrak{s}_{c}(l)\right)^{2}} \int_{0}^{l}[ & \left(\mathfrak{s}_{c}^{\prime}(r)\right)^{2}-\left(\mathfrak{s}_{c}(r)\right)^{2}\left(\mathbf{K}(\nabla r ; W)+\frac{\dot{\mathbf{S}}(\nabla r)}{n-1}\right) \\
& \left.-\frac{2 \mathfrak{s}_{c}(r) \mathfrak{s}_{c}^{\prime}(r) \mathbf{S}(\nabla r)}{n-1}\right] d r \\
\leqslant \frac{1}{\left(\mathfrak{s}_{c}(l)\right)^{2}} \int_{0}^{l}\left[\frac{N-1}{n-1} \cdot\left(\mathfrak{s}_{c}^{\prime}(r)\right)^{2}\right. & \left.\quad-\left(\mathfrak{s}_{c}(r)\right)^{2}\left(\mathbf{K}(\nabla r ; W)+\frac{\dot{\mathbf{S}}(\nabla r)}{n-1}-\frac{(\mathbf{S}(\nabla r))^{2}}{(n-1)(N-n)}\right)\right] d r
\end{aligned}
$$




$$
\leqslant \frac{N-1}{n-1} \cdot \frac{1}{\left(\mathfrak{s}_{c}(l)\right)^{2}} \int_{0}^{l}\left[\left(\mathfrak{s}_{c}^{\prime}(r)\right)^{2}-c \cdot\left(\mathfrak{s}_{c}(r)\right)^{2}\right] d r=\frac{N-1}{n-1} \cdot \mathfrak{c t}_{c}(r(q)) .
$$

Now the theorem follows easily.

Theorem 3.2 and Theorem 3.3 recommend us to introduce the notions of weighted curvatures as follows.

Definition 3.4. Let $(M, F, d \mu)$ be a Finsler $n$-manifold with volume form $d \mu$, and $y, u \in T_{x} M$ be linearly independent. For any $N \in(1, n) \cup(n, \infty)$, the weighted flag curvature is defined by

$$
\mathbf{K}_{N}(y ; u)=\mathbf{K}(y ; u)+\frac{\dot{\mathbf{S}}(y)}{(n-1) F^{2}(y)}-\frac{(\mathbf{S}(y))^{2}}{(n-1)(N-n) F^{2}(y)} .
$$

We write $\mathbf{K}_{N} \geqslant c\left(\operatorname{resp} . \mathbf{K}_{N} \leqslant c\right)$ if $\mathbf{K}_{N}(y ; u) \geqslant c\left(\operatorname{resp} . \mathbf{K}_{N}(y ; u) \leqslant c\right)$ holds for any linearly independent vectors $y, u \in T_{x} M$ and $x \in M$. Similarly, given $N \in(1, n) \cup(n, \infty)$, the weighted Ricci curvature is defined by

$$
\boldsymbol{R i c}_{N}(y)=\sum_{i=1}^{n-1} \mathbf{K}_{N}\left(y ; e_{i}\right)=\mathbf{R i c}(y)+\frac{\dot{\mathbf{S}}(y)}{F^{2}(y)}-\frac{(\mathbf{S}(y))^{2}}{(N-n) F^{2}(y)}
$$

where $e_{1}, \ldots, e_{n-1}, e_{n}=y$ is a $\mathbf{g}_{y}$-orthogonormal basis for the corresponding tangent space. We write $\mathbf{R i c} c_{N} \geqslant c$ (resp. $\mathbf{R i c} c_{N} \leqslant c$ ) if $\mathbf{R i c}_{N}(y) \geqslant c$ (resp. $\operatorname{Ric}_{N}(y) \leqslant c$ ) for any nonzero $y \in T_{x} M$ and $x \in M$.

Remark 3.5. (1) The notion of weighted Ricci curvature $\operatorname{Ric}_{N}(N \in(n, \infty))$ was first introduced by Ohta from view point of curvature-dimension condition [9]. Here we introduce the weighted Ricci curvature based on the weighted flag curvature from view point of comparison geometry. When $N \in(n, \infty)$, the notion of $\mathbf{R i c}_{N}$ above coincides with that of [9].

(2) It is clear that

$\lim _{N \rightarrow+\infty} \mathbf{K}_{N}(y ; u)=\mathbf{K}(y ; u)+\frac{\dot{\mathbf{S}}(y)}{(n-1) F^{2}(y)}, \lim _{N \rightarrow+\infty} \mathbf{R i c}_{N}(y)=\mathbf{R i c}(y)+\frac{\dot{\mathbf{S}}(y)}{F^{2}(y)}$,

thus we can define

$$
\mathbf{K}_{\infty}(y ; u)=\mathbf{K}(y ; u)+\frac{\dot{\mathbf{S}}(y)}{(n-1) F^{2}(y)}, \quad \mathbf{R i c}_{\infty}(y)=\mathbf{R i c}(y)+\frac{\dot{\mathbf{S}}(y)}{F^{2}(y)},
$$

as defined for $\mathbf{R i c} \mathbf{c}_{\infty}$ in [10]; As for the case $N=n, \lim _{N \rightarrow n} \mathbf{K}_{N}(y ; u)$ or $\lim _{N \rightarrow n} \mathbf{R i c}_{N}(y)$ cannot be finite unless $\mathbf{S}(y)=0$. In fact, when $\mathbf{S} \equiv 0$, then the weighted flag curvature and the weighted Ricci curvature are reduced to usual flag curvature and Ricci curvature for any $N \in(1, \infty)$. As a result, we may replace the weighted curvatures by usual curvatures and put $N=n$ in all results of the present paper for Finsler manifolds with vanishing S-curvature.

By using the notions of weighted curvatures we can rewrite Theorem 3.2 and Theorem 3.3 as follows. 
Theorem 3.2'. Let $(M, F, d \mu)$ and $r=d_{F}(p, \cdot)$ as above. Suppose that for some $1<N<n$, the weighted flag curvature satisfies $\mathbf{K}_{N} \leqslant \frac{N-1}{n-1} \cdot c$. Then for any vector field $X$ on $M$, the following inequality holds whenever $r$ is smooth $(r<\pi / \sqrt{c}$ when $c>0)$ :

$$
\widetilde{H}(r)(X, X) \geqslant \frac{N-1}{n-1} \cdot \mathfrak{c t}_{c}(r)\left(\mathbf{g}_{\nabla r}(X, X)-\mathbf{g}_{\nabla r}(\nabla r, X)^{2}\right) .
$$

Theorem 3.3'. Let $(M, F, d \mu)$ and $r=d_{F}(p, \cdot)$ as above. Suppose that for some $N>n$, the weighted flag curvature of $M$ satisfies $\mathbf{K}_{N} \geqslant \frac{N-1}{n-1} \cdot c$. Then, for any vector field $X$ on $M$, the following inequality holds whenever $r$ is smooth:

$$
\widetilde{H}(r)(X, X) \leqslant \frac{N-1}{n-1} \cdot \mathfrak{c t}_{c}(r)\left(\mathbf{g}_{\nabla r}(X, X)-\mathbf{g}_{\nabla r}(\nabla r, X)^{2}\right) .
$$

\section{Laplacian comparison theorems}

In this section we shall derive some Laplacian comparison theorems for distance functions. First of all, we have following result by (3.2) and Theorem 3.2 .

Theorem 4.1. Let $(M, F, d \mu)$ be a forward complete Finsler $n$-manifold, $r=$ $d_{F}(p, \cdot)$, the distance function from a fixed point $p$. Suppose that for some $1<N<n$, the weighted flag curvature of $M$ satisfies $\mathbf{K}_{N} \leqslant \frac{N-1}{n-1} \cdot c$. Then the inequality $\Delta r \geqslant(N-1) \mathfrak{c t}_{c}(r)$ holds whenever $r$ is smooth $(r<\pi / \sqrt{c}$ when $c>0)$.

Secondly, the following Laplacian comparison theorem can be verified similarly as Theorem 3.3 which was first obtained by [10].

Theorem 4.2. Let $(M, F, d \mu)$ be a forward complete Finsler n-manifold. Suppose that for some $N>n$, the weighted Ricci curvature of $M$ satisfies $\mathbf{R i c}_{N} \geqslant$ $(N-1) c$. Then $\Delta r \leqslant(N-1) \cdot \mathfrak{c t}_{c}(r)$ holds whenever $r$ is smooth.

To establish next comparison theorem, we need the following lemma.

Lemma 4.3. Let $X$ be a local vector field on an open set $\mathcal{U}$ of $p \in M$ with $\mathbf{g}_{\nabla r}(X, X)=1, \mathbf{g}_{\nabla r}(\nabla r, X)=0$. Then $\widetilde{H}(r)(X, X) \sim \frac{1}{r}$ as $r \rightarrow 0^{+}$.

Proof. Let $b$ and $c$ be the lower bound and upper bound of flag curvature on $\overline{\mathcal{U}}$, respectively. Then by Hessian comparison theorem [17] we have

$$
\mathfrak{c t}_{c}(r) \leqslant H(r)(X, X) \leqslant \mathfrak{c t}_{b}(r) .
$$

Note that $\widetilde{H}(r)(X, X)=H(r)(X, X)-\frac{\mathbf{S}(\nabla r)}{n-1}$ and $\mathfrak{c t}_{c}(r) \sim \frac{1}{r}$ as $r \rightarrow 0^{+}$for any $c$, we easily get the result.

Now we are ready to prove the following Laplacian comparison theorem in terms of upper bounds on the weighted Ricci curvature. 
Theorem 4.4. Let $(M, F, d \mu)$ be a forward complete Finsler n-manifold with nonpositive flag curvature. Suppose that the weighted Ricci curvature satisfies $\mathbf{R i c}_{N} \leqslant(N+1-n) c<0$ for some $n-1<N<n$. Then $\Delta r \geqslant(N+1-n) \mathfrak{c t}_{c}(r)$ holds whenever $r$ is smooth.

Proof. Suppose that $r$ is smooth at $q \in M$, and $\gamma:[0, r(q)] \rightarrow M$ be the unique minimal geodesic from $p$ to $q$. We choose the local $\mathbf{g}_{\nabla r}$-orthonormal frame $E_{1}, \ldots, E_{n-1}, E_{n}=\nabla r$ that is parallel along $\gamma$, then by (2.1)-(2.4) we have

$$
\begin{aligned}
& \frac{d}{d r}\left(H(r)\left(E_{i}, E_{j}\right)\right)=\frac{d}{d r} \mathbf{g}_{\nabla r}\left(\nabla E_{i} \nabla r, E_{j}\right) \\
& =\mathbf{g}_{\nabla r}\left(\nabla \underset{\nabla r}{\nabla r} \nabla E_{i}^{\nabla r} \nabla r, E_{j}\right) \\
& =\mathbf{g}_{\nabla r}\left(\mathbf{R}^{\nabla r}\left(\nabla r, E_{i}\right) \nabla r, E_{j}\right)+\mathbf{g}_{\nabla r}\left(\nabla_{\left[\nabla r, E_{i}\right]}^{\nabla r} \nabla r, E_{j}\right) \\
& =-\mathbf{g}_{\nabla r}\left(\mathbf{R}^{\nabla r}\left(E_{i}, \nabla r\right) \nabla r, E_{j}\right)-\mathbf{g}_{\nabla r}\left(\nabla_{\nabla_{E_{i}}^{\nabla r} \nabla r}^{\nabla r} \nabla r, E_{j}\right) \\
& =-\mathbf{g}_{\nabla r}\left(\mathbf{R}^{\nabla r}\left(E_{i}, \nabla r\right) \nabla r, E_{j}\right)-\sum_{k} \mathbf{g}_{\nabla r}\left(\nabla E_{i} r \nabla r, E_{k}\right) \mathbf{g}_{\nabla r}\left(\nabla_{E_{k}}^{\nabla r} \nabla r, E_{j}\right) \\
& =-\mathbf{g}_{\nabla r}\left(\mathbf{R}^{\nabla r}\left(E_{i}, \nabla r\right) \nabla r, E_{j}\right)-\sum_{k} H(r)\left(E_{i}, E_{k}\right) \cdot H(r)\left(E_{k}, E_{j}\right),
\end{aligned}
$$

and thus

$$
\frac{d}{d r}\left(\operatorname{tr}_{\nabla r} H(r)\right)=-\mathbf{R i c}(\nabla r)-\sum_{i, j}\left(H(r)\left(E_{i}, E_{j}\right)\right)^{2}
$$

Now since $M$ has nonpositive flag curvature, by Hessian comparison theorem [17] we see that the eigenvalues of $\left(H(r)\left(E_{i}, E_{j}\right)\right)_{1 \leqslant i, j \leqslant n-1}$ are all positive, which together with (4.1) implies that

$$
\frac{d}{d r}\left(\operatorname{tr}_{\nabla r} H(r)\right) \geqslant-\mathbf{R i c}(\nabla r)-\left(\operatorname{tr}_{\nabla r} H(r)\right)^{2} .
$$

Consequently,

$$
\frac{d}{d r} \Delta r \geqslant-\mathbf{R i c}(\nabla r)-\dot{\mathbf{S}}(\nabla r)-(\Delta r+\mathbf{S}(\nabla r))^{2} .
$$

Furthermore we have

$$
2 \Delta r \cdot \mathbf{S}(\nabla r) \leqslant \frac{N+1-n}{n-N}(\mathbf{S}(\nabla r))^{2}+\frac{n-N}{N+1-n}(\Delta r)^{2} .
$$

Combining (4.2) and (4.3) it is easy to get

$$
\frac{d}{d r} \Delta r \geqslant-\operatorname{Ric}_{N}(\nabla r)-\frac{1}{N+1-n}(\Delta r)^{2},
$$

which together with $\mathbf{R i c}_{N} \leqslant(N+1-n) c$ yields

$$
\frac{d}{d r}\left(\frac{1}{N+1-n} \Delta r\right) \geqslant-c-\left(\frac{1}{N+1-n} \Delta r\right)^{2} \text {. }
$$


Note that

$$
\frac{d}{d r} \mathfrak{c t}_{c}(r)=-c-\left(\mathfrak{c t}_{c}(r)\right)^{2}
$$

we have

$$
\frac{d}{d r}\left(\frac{1}{N+1-n} \Delta r-\mathfrak{c t}_{c}(r)\right) \geqslant-\left(\frac{1}{N+1-n} \Delta r\right)^{2}+\left(\mathfrak{c t}_{c}(r)\right)^{2} .
$$

Putting

$$
A(r)=\frac{1}{N+1-n} \Delta r-\mathfrak{c t}_{c}(r), \quad B(r)=\frac{1}{N+1-n} \Delta r+\mathfrak{c t}_{c}(r),
$$

then (4.4) becomes

$$
\frac{d A}{d r}+A B \geqslant 0
$$

By Lemma 4.3 we see that $A(r) \rightarrow+\infty$ as $r \rightarrow 0^{+}$, thus there is $\epsilon>0$ such that $A(r)>0, \forall r \in(0, \epsilon]$. On the other hand, from (4.5) we have

$$
\frac{d}{d r}\left(A(r) \exp \left(\int_{\epsilon}^{r} B(s) d s\right)\right) \geqslant 0
$$

which yields

Therefore,

$$
A(r) \exp \left(\int_{\epsilon}^{r} B(s) d s\right) \geqslant A(\epsilon) \geqslant 0
$$

$$
\Delta r \geqslant(N+1-n) \mathfrak{c t}_{c}(r) .
$$

\section{Volume comparison theorems}

In this section we shall use the Laplacian comparison theorems to derive some volume comparison theorems for Finsler manifolds.

Let $(M, F, d \mu)$ be a Finsler manifold. Fix $p \in M$, let $I_{p}=\left\{v \in T_{p} M\right.$ : $F(v)=1\}$ be the indicatrix at $p$. For $v \in I_{p}$, the cut-value $c(v)$ is defined by

$$
c(v):=\sup \left\{t>0: d_{F}\left(p, \exp _{p}(t v)\right)=t\right\} .
$$

Then, we can define the tangential cut locus $\mathbf{C}(p)$ of $p$ by $\mathbf{C}(p):=\{c(v) v$ : $\left.c(v)<\infty, v \in I_{p}\right\}$, the cut locus $C(p)$ of $p$ by $C(p)=\exp _{p} \mathbf{C}(p)$, and the injectivity radius $i_{p}$ at $p$ by $i_{p}=\inf \left\{c(v): v \in I_{p}\right\}$, respectively. It is known that $C(p)$ has zero Hausdorff measure in $M$. Also, we set $\mathbf{D}_{p}=\{t v: 0 \leqslant t<$ $\left.c(v), v \in I_{p}\right\}$ and $D_{p}=\exp _{p} \mathbf{D}_{p}$. Let $B_{p}(R)$ be the forward geodesic ball of $M$ with radius $R$ centered at $p$. The volume of $B_{p}(R)$ with respect to $d \mu$ is defined by

$$
\operatorname{vol}\left(B_{p}(R)\right)=\int_{B_{p}(R)} d \mu .
$$

In order to compute the volume, we need polar coordinates on $D_{p}$. Let $\theta^{\alpha}$, $\alpha=1, \ldots, n-1$ be the local coordinates that are intrinsic to $I_{p}$. For any $q \in D_{p}$, the polar coordinates of $q$ are defined by $(r, \theta)=\left(r(q), \theta^{1}(q), \ldots, \theta^{n-1}(q)\right)$, where $r(q)=F(v), \theta^{\alpha}(q)=\theta^{\alpha}\left(\frac{v}{F(v)}\right)$, and $v=\exp _{p}^{-1}(q)$. Writing $d \mu=$ 
$\sigma(r, \theta) d r \wedge d \theta^{1} \wedge \cdots \wedge \theta^{n-1}:=\sigma(r, \theta) d r \wedge d \theta$, by the by the definition of Laplacian we have (see [17])

$$
\Delta r=\frac{\partial}{\partial r} \log \sigma
$$

For $r>0$, let $\mathbf{D}_{p}(r) \subset I_{p}$ be defined by

$$
\mathbf{D}_{p}(r)=\left\{v \in I_{p}: r v \in \mathbf{D}_{p}\right\} .
$$

It is easy to see that $\mathbf{D}_{p}\left(r_{1}\right) \subset \mathbf{D}_{p}\left(r_{2}\right)$ for $r_{1}>r_{2}$ and $\mathbf{D}_{p}(r)=I_{p}$ for $r<i_{p}$. Since $C(p)$ has zero Hausdorff measure in $M$, we have

$$
\begin{aligned}
\operatorname{vol}\left(B_{p}(R)\right) & =\int_{B_{p}(R)} d \mu=\int_{B_{p}(R) \cap D_{p}} d \mu \\
& =\int_{\exp _{p}^{-1}\left(B_{p}(R)\right) \cap \mathbf{D}_{p}} \exp _{p}^{*}(d \mu)=\int_{0}^{R} d r \int_{\mathbf{D}_{p}(r)} \sigma(r, \theta) d \theta .
\end{aligned}
$$

For real numbers $c$ and $\Lambda$, let

$$
V_{c, \Lambda}(r)=\int_{0}^{r} \mathfrak{s}_{c}(t)^{\Lambda-1} d t .
$$

Now we are ready to prove the following:

Theorem 5.1. Let $(M, F, d \mu)$ be a forward complete Finsler n-manifold which satisfies $\mathbf{K}_{N} \leqslant \frac{N-1}{n-1} \cdot c$ for some $1<N<n$. Then the function

$$
\frac{\operatorname{vol}\left(B_{p}(r)\right)}{V_{c, N}(r)}
$$

is monotone increasing for $0<r \leqslant i_{p}$, where $i_{p}$ is the injectivity radius of $p$.

Proof. By (5.1), Theorem 4.1 and the assumptions of the theorem, we have

$$
\frac{\partial}{\partial r} \log \sigma \geqslant(N-1) \mathfrak{c t}_{c}(r)=\frac{d}{d r} \log \mathfrak{s}_{c}(r)^{N-1},
$$

thus the function

$$
\frac{\int_{I_{p}} \sigma(r, \theta) d \theta}{\mathfrak{s}_{c}(r)^{N-1}}
$$

is monotone increasing about $r\left(\leqslant i_{p}\right)$. Now by the standard argument [4], the function

$$
\frac{\int_{0}^{r} \int_{I_{p}} \sigma(t, \theta) d t d \theta}{\int_{0}^{r} \mathfrak{s}_{c}(t)^{N-1} d t}=\frac{\operatorname{vol}\left(B_{p}(r)\right)}{V_{c, N}(r)}
$$

is also monotone increasing for $R \leqslant i_{p}$.

The following theorem can be shown similarly using Theorem 4.4. 
Theorem 5.2. Let $(M, F, d \mu)$ be a complete and simply connected Finsler $n$-manifold with nonpositive flag curvature. If the weighted Ricci curvature satisfies $\mathbf{R i c}_{N} \leqslant(N+1-n) c<0$ for some $n-1<N<n$, then the function

$$
\frac{\operatorname{vol}\left(B_{p}(r)\right)}{V_{c, N+2-n}(r)}
$$

is monotone increasing.

The following theorem was first obtained in [9], and here we provide another proof using Laplacian comparison theorem.

Theorem 5.3. Let $(M, F, d \mu)$ be a forward complete Finsler n-manifold. Suppose that for some $N>n$, the weighted Ricci curvature of $M$ satisfies $\mathbf{R i c}_{N} \geqslant$ $(N-1) c$. Then the function

$$
\frac{\operatorname{vol}\left(B_{p}(r)\right)}{V_{c, N}(r)}
$$

is monotone decreasing in $r$.

Proof. By (5.1), Theorem 4.2 and the assumptions of the theorem, we have

thus the function

$$
\frac{\partial}{\partial r} \log \sigma \leqslant(N-1) \mathfrak{c t}_{c}(r)=\frac{d}{d r} \log \mathfrak{s}_{c}(r)^{N-1},
$$

$$
\frac{\sigma(r, \theta)}{\mathfrak{s}_{c}(r)^{N-1}}
$$

is monotone decreasing about $r$. Noting that $\mathbf{D}_{p}(R) \subset \mathbf{D}_{p}(r)$ for $R>r>0$, we have for $R>r>0$,

$$
\begin{aligned}
\frac{\int_{\mathbf{D}_{p}(r)} \sigma(r, \theta) d \theta}{\mathfrak{s}_{c}(r)^{N-1}} & =\int_{\mathbf{D}_{p}(r)} \frac{\sigma(r, \theta)}{\mathfrak{s}_{c}(r)^{N-1}} d \theta \geqslant \int_{\mathbf{D}_{p}(R)} \frac{\sigma(r, \theta)}{\mathfrak{s}_{c}(r)^{N-1}} d \theta \\
& \geqslant \int_{\mathbf{D}_{p}(R)} \frac{\sigma(R, \theta)}{\mathfrak{s}_{c}(R)^{N-1}} d \theta=\frac{\int_{\mathbf{D}_{p}(R)} \sigma(R, \theta) d \theta}{\mathfrak{s}_{c}(R)^{N-1}}
\end{aligned}
$$

namely,

$$
\frac{\int_{\mathbf{D}_{p}(r)} \sigma(r, \theta) d \theta}{\mathfrak{s}_{c}(r)^{N-1}}
$$

is also monotone decreasing. Now the theorem can be verified easily.

A theorem due to Calabi and Yau states that the volume of any complete noncompact Riemannian manifold with nonnegative Ricci curvature has at least linear growth $[3,18]$. This result has been generalized to Finsler manifolds by [15], where we establish a similar result in terms of weighted Ricci curvature. For this purpose we need the notion of reversibility for Finsler manifolds. For a given Finsler manifold $(M, F)$, the reversibility $\lambda_{F}$ of $(M, F)$ is defined by (see [11])

$$
\lambda_{F}=\max _{X \in T M \backslash 0} \frac{F(X)}{F(-X)} .
$$


$(M, F)$ is called reversible if $\lambda_{F}=1$. It is clear that the induced distance function $d_{F}$ of $F$ satisfies

$$
d_{F}(p, q) \leqslant \lambda_{F} d_{F}(q, p), \quad \forall p, q \in M
$$

Now we can prove:

Theorem 5.4. Let $(M, F, d \mu)$ be a complete noncompact Finsler $n$-manifold with $\mathbf{R i c}_{N} \geqslant 0$ for some $N>n$, then $M$ must have infinite volume. Furthermore, if $M$ has finite reversibility, then the volume $\operatorname{vol}\left(B_{p}(R)\right)$ of the forward geodesic ball has at least linear growth:

$$
\operatorname{vol}\left(B_{p}(R)\right) \geqslant c(p) R
$$

Proof. Since $M$ is complete and noncompact, there is a geodesic $\gamma:(-\infty, 0] \rightarrow$ $M$ such that $\gamma(0)=p, d_{F}\left(\gamma\left(-t_{2}\right), \gamma\left(-t_{1}\right)\right)=t_{2}-t_{1}, \forall t_{2}>t_{1}>0$. Denote by $B_{p}^{-}(r)=\left\{x \in M: d_{F}(x, p)<r\right\}$ be the backward geodesic ball of radius $r$ centered at $p$, then by the triangle inequality it is easy to see that

$$
B_{p}(1) \cap B_{p}^{-}(1) \subset B_{\gamma(-t)}(t+1) \backslash B_{\gamma(-t)}(t-1), \quad \forall t>1 .
$$

Since $\mathbf{R i c}_{N} \geqslant 0$ for some $N>n$, by Theorem 5.3 we have

$$
\frac{\operatorname{vol}\left(B_{\gamma(-t)}(R)\right)}{R^{N}} \leqslant \frac{\operatorname{vol}\left(B_{\gamma(-t)}(r)\right)}{r^{N}}, \quad \forall r<R,
$$

and consequently,

$$
\operatorname{vol}\left(B_{\gamma(-t)}(r)\right) \geqslant \frac{r^{N}}{R^{N}-r^{N}}\left(\operatorname{vol}\left(B_{\gamma(-t)}(R)\right)-\operatorname{vol}\left(B_{\gamma(-t)}(r)\right)\right) .
$$

(5.2) and (5.3) yields

$$
\begin{aligned}
\operatorname{vol}\left(B_{\gamma(-t)}(t-1)\right) & \geqslant \frac{(t-1)^{N}}{(t+1)^{N}-(t-1)^{N}}\left(\operatorname{vol}\left(B_{\gamma(-t)}(t+1)\right)-\operatorname{vol}\left(B_{\gamma(-t)}(t-1)\right)\right) \\
& \geqslant \frac{(t-1)^{N}}{(t+1)^{N}-(t-1)^{N}} \operatorname{vol}\left(B_{p}(1) \cap B_{p}^{-}(1)\right) .
\end{aligned}
$$

Since

$$
\lim _{t \rightarrow+\infty} \frac{(t-1)^{N}}{t\left((t+1)^{N}-(t-1)^{N}\right)}=\frac{1}{2 N}
$$

there is a constant $\delta>0$ such that

and thus

$$
\frac{(t-1)^{N}}{(t+1)^{N}-(t-1)^{N}} \geqslant \delta t, \quad \forall t>1
$$

$$
\operatorname{vol}\left(B_{\gamma(-t)}(t-1)\right) \geqslant \delta t \cdot \operatorname{vol}\left(B_{p}(1) \cap B_{p}^{-}(1)\right) .
$$

It follows that $M$ has infinite volume. Furthermore, if $M$ has finite reversibility $\lambda_{F}$, then again by the triangle inequality we easily see that

$$
B_{\gamma(-t)}(t-1) \subset B_{\gamma(-t)}^{-}\left(\lambda_{F}(t-1)\right) \subset B_{p}^{-}\left(2 \lambda_{F} t\right) \subset B_{p}\left(2 \lambda_{F}^{2} t\right),
$$


and which together with (5.4) yields

$$
\operatorname{vol}\left(B_{p}\left(2 \lambda_{F}^{2} t\right)\right) \geqslant \delta t \cdot \operatorname{vol}\left(B_{p}(1) \cap B_{p}^{-}(1)\right):=c(p) \cdot 2 \lambda_{F}^{2} t,
$$

where $c(p)$ is a constant depending on $p$. Letting $R=2 \lambda_{F}^{2} t$ we obtain the desired inequality.

Remark 5.5. The second conclusion of Theorem 5.4 has been verified recently in [19] by a different argument.

\section{The first eigenvalue}

In this section we shall study the first eigenvalue on Finsler manifolds with weighted bounded curvature. Let $(M, F, d \mu)$ be a Finsler $n$-manifold, $\Omega \subset M$ a domain with compact closure and nonempty boundary $\partial \Omega$. The first eigenvalue $\lambda_{1}(\Omega)$ of $\Omega$ is defined by (see [13], page 176)

$$
\lambda_{1}(\Omega)=\inf _{f \in L_{1,0}^{2}(\Omega) \backslash\{0\}}\left\{\frac{\int_{\Omega}\left(F^{*}(d f)\right)^{2} d \mu}{\int_{\Omega} f^{2} d \mu}\right\},
$$

where $L_{1,0}^{2}(\Omega)$ is the completion of $C_{0}^{\infty}(\Omega)$. If $\Omega_{1} \subset \Omega_{2}$ are bounded domains, then $\lambda_{1}\left(\Omega_{1}\right) \geqslant \lambda_{1}\left(\Omega_{2}\right) \geqslant 0$. Thus, if $\Omega_{1} \subset \Omega_{2} \subset \cdots \subset M$ be bounded domains so that $\bigcup \Omega_{i}=M$, then the following limit

$$
\lambda_{1}(M)=\lim _{i \rightarrow \infty} \lambda_{1}\left(\Omega_{i}\right) \geqslant 0
$$

exists, and it is independent of the choice of $\left\{\Omega_{i}\right\}$. The following lemma is crucial in this section.

Lemma 6.1 ([17]). Let $(M, F, d \mu)$ be a Finsler manifold with finite reversibility $\lambda_{F}, \Omega \subset M$ a domain with compact closure and nonempty boundary, and $X$ a vector field on $\Omega$ so that $\|X\|_{\infty}=\sup _{\Omega} F(X)<\infty$ and $\inf _{\Omega} \operatorname{div}(X)>0$. Then

$$
\lambda_{1}(\Omega) \geqslant\left[\frac{\inf _{\Omega} \operatorname{div} X}{2 \lambda_{F}\|X\|_{\infty}}\right]^{2} .
$$

By Lemma 6.1 we can prove:

Theorem 6.2. Let $(M, F, d \mu)$ be a forward complete Finsler n-manifold with finite reversibility $\lambda_{F}$. Let $B_{p}(R)$ be the forward geodesic ball of $M$ with radius $R$ centered at $p$, and $R<i_{p}$, where $i_{p}$ denotes the injectivity radius about $p$. We have:

(1) if the weighted flag curvature satisfies $\mathbf{K}_{N} \leqslant \frac{N-1}{n-1} \cdot c<0$ for some $1<N<n$, then

$$
\lambda_{1}\left(B_{p}(R)\right) \geqslant \frac{(N-1)^{2}\left(\mathfrak{c t}_{c}(R)\right)^{2}}{4 \lambda_{F}^{2}}
$$

(2) if $M$ has nonpositive flag curvature and the weighted Ricci curvature satisfies $\mathbf{R i c}_{N} \leqslant(N+1-n) c<0$ for some $n-1<N<n$, then

$$
\lambda_{1}\left(B_{p}(R)\right) \geqslant \frac{(N+1-n)^{2}\left(\mathfrak{c t}_{c}(R)\right)^{2}}{4 \lambda_{F}^{2}} .
$$


Proof. We shall only prove (1), (2) can be verified similarly. For $R>\epsilon>0$, let $\Omega_{\epsilon}=B_{p}(R) \backslash \overline{B_{p}(\epsilon)}$. Then $r=d_{F}(p, \cdot)$ is smooth on $\Omega_{\epsilon}$, and thus $X=\nabla r$ is a smooth vector field on $\Omega_{\epsilon}$. Noting that $F(X)=F(\nabla r)=1$ and $\operatorname{div} X=\Delta r$, we deduce from Theorem 4.1 and Lemma 6.1 that

$$
\lambda_{1}\left(\Omega_{\epsilon}\right) \geqslant \frac{(N-1)^{2}\left(\mathfrak{c t}_{c}(R)\right)^{2}}{4 \lambda_{F}^{2}} .
$$

Letting $\epsilon \rightarrow 0$, we get the desired inequality.

Mckean [7] proved that if $(M, g)$ is a complete and simply connected Riemannian $n$-manifold with sectional curvature $\mathbf{K} \leqslant-a^{2}$, then $\lambda_{1}(M) \geqslant \frac{(n-1)^{2} a^{2}}{4}$. Afterwards, this result was extended by Ding in [5] for Riemannian manifolds with nonpositive curvature and Ricci curvature bounded from above by a negative constant. These results has been generalized into Finsler manifolds in $[15,17]$, and by Theorem 6.2 we have the following result in terms of weighted curvatures.

Theorem 6.3. Let $(M, F, d \mu)$ be a forward complete and simply connected Finsler n-manifold with finite reversibility $\lambda_{F}$ and nonpositive flag curvature. We have:

(1) if the weighted flag curvature satisfies $\mathbf{K}_{N} \leqslant-\frac{N-1}{n-1} a^{2}(a>0)$ for some $1<N<n$, then

$$
\lambda_{1}(M) \geqslant \frac{(N-1)^{2} a^{2}}{4 \lambda_{F}^{2}}
$$

(2) if the weighted Ricci curvature satisfies $\mathbf{R i c}_{N} \leqslant-(N+1-n) a^{2}(a>0)$ for some $n-1<N<n$, then

$$
\lambda_{1}(M) \geqslant \frac{(N+1-n)^{2} a^{2}}{4 \lambda_{F}^{2}} .
$$

\section{Weighted curvature and fundamental group}

In this section we shall study the relationship between weighted curvature and fundamental group of Finsler manifolds. Let us first recall some basic facts about universal covering space and fundamental group. Let $(M, F)$ be a Finsler $n$-manifold, and $f: \widetilde{M} \rightarrow M$ be the universal covering space. A homeomorphism $\varphi: \widetilde{M} \rightarrow \widetilde{M}$ is called a deck transformation of the coving mapping $f$ if $f \circ \varphi=f$. The set of deck transformations $\Gamma$ obviously form a group nuder composition. One checks that the deck transformation group $\Gamma$ acts properly discontinuously on $\widetilde{M}$. If we endow the pulled-back metric $\widetilde{F}=f^{*} F$ on $\widetilde{M}$, then $f:(\widetilde{M}, \widetilde{F}) \rightarrow(M, F)$ is a local isometry, and it is easy to check that each $\gamma \in \Gamma$ is an isometry, and $(M, F)$ and $(\widetilde{M}, \widetilde{F})$ have the same reversibility and uniformity constant. It is also clear that if $(M, F)$ is (forward) complete, then so is $(\widetilde{M}, \widetilde{F})$ (see [14]). 
Given $p \in M$, let $\pi_{1}(M, p)$ be the fundamental group of $M$ based at $p$, namely, the homotopy classes of loops $\gamma:[0,1] \rightarrow M \in C^{0}$ satisfying $\gamma(0)=$ $\gamma(1)=p$. It is well-known that the deck transformation group $\Gamma$ is isomorphic to $\pi_{1}(M, p)$, and $\Gamma$ acts transitively on $f^{-1}(p)$ for each $p \in M[4]$. In the following, we shall identify $\pi_{1}(M, p)$ with the deck transformation group $\Gamma$. Given any point $p \in M$, for each $\gamma \in \Gamma \cong \pi_{1}(M, p)$, the geometric norm $\|\gamma\|$ associated with $p$ is defined by

$$
\|\gamma\|=d_{\widetilde{F}}(\widetilde{p}, \gamma(\widetilde{p}))
$$

where $\widetilde{p}$ is any point in the fiber $f^{-1}(p)$, and $d_{\widetilde{F}}$ is the distance function on $\widetilde{M}$ induced by $\widetilde{F}$. It is known that the geometric norm $\|\gamma\|$ equals the length of a shortest loop representing $\gamma \in \pi_{1}(M, p)$, which is a geodesic loop. Also, the set $\Delta(\lambda)=\{\gamma \in \Gamma:\|\gamma\| \leqslant \lambda\}$ is finite for any $\lambda>0$, and it is natural to propose the following definition $[16]$.

Definition 7.1. The counting function $N(\lambda)$ of the fundamental group $\Gamma \cong$ $\pi_{1}(M, p)$ of $(M, F)$ is defined by

$$
N(\lambda)=\sharp \Delta(\lambda)=\sharp\{\gamma \in \Gamma:\|\gamma\| \leqslant \lambda\} .
$$

$\Gamma$ is said to have exponential growth if

$$
\limsup _{\lambda \rightarrow \infty} \frac{\log N(\lambda)}{\lambda}>0 .
$$

$\Gamma$ is said to have polynomial growth of order $\leqslant \alpha$ if $N(\lambda) \leqslant$ constant $\cdot \lambda^{\alpha}$.

Remark 7.2. Let $\Gamma^{\prime} \subset \pi_{1}(M, p)$ be any finitely generated subgroup with a set of generators $S=\left\{\gamma_{1}, \ldots, \gamma_{k}\right\}$. The counting function $n(\lambda)$ of $\Gamma^{\prime}$ considered in $[1,8,14]$ is defined by

$$
n(\lambda)=\sharp\left\{\gamma \in \Gamma^{\prime}:|\gamma| \leqslant \lambda\right\},
$$

where $|\gamma|$ is the minimum length of $\gamma$ as a word in $\left\{\gamma_{1}, \ldots, \gamma_{k}\right\}$. The advantage of our definition is that it does not demand that $\Gamma$ is finitely generated.

The following lemma is crucial to study weighted curvature and fundamental group, it can be verified by using the fundamental domain of covering mapping [16].

Lemma 7.3. Let $(M, F)$ be a forward complete Finsler manifold and $f$ : $(\widetilde{M}, \widetilde{F}) \rightarrow(M, F)$ denote its universal covering projection with deck transformation group $\Gamma$. Fix $p \in M$, and $\widetilde{p} \in f^{-1}(p)$. Then

(1) the counting function of $\Gamma$ satisfies

$$
N(\lambda) \leqslant \frac{\operatorname{vol}\left(\widetilde{B}_{\widetilde{p}}(\lambda+R)\right)}{\operatorname{vol}\left(B_{p}(R)\right)}, \quad \forall \lambda>0, R>0
$$


(2) if $(M, F)$ has finite reversibility, namely, $\lambda_{F}:=\max _{x \in M} \lambda(x)<\infty$, then

$$
N(\lambda) \geqslant \frac{\operatorname{vol}\left(\widetilde{B}_{\widetilde{p}}\left(\frac{\lambda}{1+\lambda_{F}}\right)\right)}{\operatorname{vol}\left(B_{p}\left(\frac{\lambda}{1+\lambda_{F}}\right)\right)},
$$

where $\widetilde{B}_{\widetilde{p}}(R)$ is the forward geodesic ball in $(\widetilde{M}, \widetilde{F})$ centered at $\widetilde{p}$ with radius $R$.

Now we can prove:

Theorem 7.4. Let $(M, F, d \mu)$ be a forward complete Finsler n-manifold with weighted Ricci curvature satisfying $\mathbf{R i c}_{N} \geqslant 0$ for some $N>n$. If there exists $p \in M$ such that $\operatorname{vol}\left(B_{p}(R)\right) \geqslant C \cdot R^{k}$ for some constant $C$ and $0 \leqslant k \leqslant N$, then $\pi_{1}(M)$ has polynomial growth of order $\leqslant N-k$. In particular, the fundamental group of any forward complete noncompact Finsler manifold with weighted Ricci curvature satisfying $\mathbf{R i c}_{N} \geqslant 0$ for some $N>n$ and finite reversibility must have polynomial growth of order $\leqslant N-1$.

Proof. Fix $\widetilde{p} \in f^{-1}(p)$. Then we have by Theorem 5.3 ,

$$
\frac{\operatorname{vol}\left(B_{p}(R)\right)}{R^{N}} \leqslant \operatorname{vol}\left(B_{p}(1)\right), \quad \forall R>1,
$$

which together with (7.1) yields

$$
N(R) \leqslant \frac{\operatorname{vol}\left(\widetilde{B}_{\widetilde{p}}(2 R)\right)}{\operatorname{vol}\left(B_{p}(R)\right)} \leqslant \frac{2^{N} \operatorname{vol}\left(B_{p}(1)\right)}{C} \cdot R^{N-k} .
$$

Thus $\Gamma \cong \pi_{1}(M)$ has polynomial growth of order $\leqslant N-k$. Now the second assertion easily follows from Theorem 5.4.

Theorem 7.5. Let $(M, F, d \mu)$ be a forward complete Finsler n-manifold with nonpositive flag curvature and finite reversibility. Suppose one of the following two conditions holds:

(1) the weighted flag curvature of $M$ satisfies $\mathbf{K}_{N} \leqslant-\frac{N-1}{n-1} \cdot a^{2}$ for some $1<N<n$, and $\operatorname{vol}\left(B_{p}(R)\right) \leqslant c \cdot \exp ((N-1) b R)$ for some $p \in M, c>0$ and $0<b<a$;

(2) the weighted Ricci curvature satisfies $\mathbf{R i c}_{N} \leqslant-(N+1-n) a^{2}$ for some $n-1<N<n$, and $\operatorname{vol}\left(B_{p}(R)\right) \leqslant c \cdot \exp ((N+1-n) b R)$ for some $p \in M$, $c>0$ and $0<b<a$.

Then $\pi_{1}(M)$ has exponential growth.

Proof. We shall only prove (1), (2) can be verified similarly. It is clear by the curvature assumption that the injectivity radius of the universal covering space $(\widetilde{M}, \widetilde{F})$ is infinite, thus by Theorem 6.1 we have

$$
\frac{\operatorname{vol}\left(\widetilde{B}_{\widetilde{p}}(R)\right)}{V_{-a^{2}, N}(R)} \geqslant \frac{\operatorname{vol}\left(\widetilde{B}_{\widetilde{p}}(1)\right)}{V_{-a^{2}, N}(1)}:=d, \quad \forall R>1,
$$


which together with (7.2) yields

$$
\begin{aligned}
\frac{\log N(\lambda)}{\lambda} & \geqslant \frac{\log \left(\operatorname{vol}\left(\widetilde{B}_{\widetilde{p}}\left(\frac{\lambda}{1+\lambda_{F}}\right)\right)\right)}{\lambda}-\frac{\log \left(\operatorname{vol}\left(B_{p}\left(\frac{\lambda}{1+\lambda_{F}}\right)\right)\right)}{\lambda} \\
& \geqslant \frac{\log V_{-a^{2}, N}\left(\frac{\lambda}{1+\lambda_{F}}\right)}{\lambda}-\frac{(N-1) b}{1+\lambda_{F}}+\frac{\log d-\log c}{\lambda}, \quad \forall \lambda>1+\lambda_{F} .
\end{aligned}
$$

Recall that

$$
\begin{aligned}
V_{-a^{2}, N}(R) & =\int_{0}^{R}\left(\frac{\sinh a t}{a}\right)^{N-1} d t \\
& >\frac{1}{2^{N-1} a^{N-1}} \int_{1}^{R} \exp ((N-1) a t)(1-\exp (-2 a t))^{N-1} d t \\
& >\frac{(1-\exp (-2 a))^{N-1}}{(N-1) 2^{N-1} a^{N}}[\exp ((N-1) a R)-\exp ((N-1) a)]
\end{aligned}
$$

for all $R>1$, we have

$$
\frac{\log N(\lambda)}{\lambda} \geqslant \frac{(N-1)(a-b)}{1+\lambda_{F}}+\frac{C}{\lambda}, \quad \forall \lambda>1+\lambda_{F},
$$

where $C$ is a constant. Hence,

$$
\limsup _{\lambda \rightarrow \infty} \frac{\log N(\lambda)}{\lambda} \geqslant \frac{(N-1)(a-b)}{1+\lambda_{F}}>0,
$$

namely, $\pi_{1}(M)$ has exponential growth.

Remark 7.6. Theorem 7.4 and Theorem 7.5 can be viewed as weighted curvature version of corresponding results of [16]. Since we do not demand that the fundamental group is finitely generated or the manifold is compact, they are new even for Riemannian manifolds.

\section{Gromov simplicial norms}

In this last section we shall provide an upper bound for Gromov simplicial norms of reversible Finsler manifolds in terms of weighted Ricci curvature. For this purpose, let us first recall the notion of Gromov simplicial norms $[6,13]$. Let $M$ be a topological space, and $C_{k}(M)$ the $k$-th complex of real singular chains $c=\sum_{i} r_{i} \sigma_{i}$, where $\sigma_{i}: \Delta^{k} \rightarrow M$ are $k$-dimensional simplices and $r_{i}$ are the real numbers which are all, but finite, zero. Denote by $H_{k}(M)$, the real singular homology group of $M$. The $L^{1}$ norm on $C_{k}(M)$ is defined by

$$
\|c\|_{1}=\sum_{i}\left|r_{i}\right|, \quad \forall c=\sum_{i} r_{i} \sigma_{i} \in C_{k}(M) .
$$

$\|\cdot\|_{1}$ induces a pseudo-norm $\|\cdot\|$ in $H_{k}(M)$ as follows. For a class $z \in H_{k}(M)$, $\|z\|$ is given by

$$
\|z\|:=\inf _{z=[c]}\|c\|_{1} .
$$


$\|\cdot\|$ is called the Gromov simplicial norm. Assume that $M$ is an $n$-dimensional closed oriented manifold, for the fundamental class $[M] \in H_{n}(M)$, we set $\|M\|:=\|[M]\|$. The constant $\|M\|$ is called the Gromov simplicial volume. Gromov prove the following

Lemma 8.1 ([6, 13]). Let $(M, F)$ be an $n$-dimensional closed oriented reversible Finsler manifold, and $\pi:(\widetilde{M}, \widetilde{F}) \rightarrow(M, F)$ the universal covering space with pulled back metric. Then, for any $z \in H_{k}(M)$,

$$
\|z\| \leqslant k ! \min _{r>0} \sup _{\widetilde{x} \in \widetilde{M}}\left[\frac{A\left(S_{\widetilde{x}}(r)\right)}{\operatorname{vol}_{B H}\left(B_{\widetilde{x}}(r)\right)}\right]^{k} \operatorname{vol}_{B H}(z),
$$

where $B_{\widetilde{x}}(r)$ and $S_{\widetilde{x}}(r)$ are the geodesic ball and geodesic sphere of $(\widetilde{M}, \widetilde{F})$ with radius $r$ centered at $\widetilde{x}$ respectively, $\operatorname{vol}_{B H}$ is the volume about to the BusemannHausdorff volume form, and $A\left(S_{\widetilde{x}}(r)\right)=\frac{d}{d r} \operatorname{vol}_{B H}\left(B_{\widetilde{x}}(r)\right)$. In particular,

$$
\|M\| \leqslant n ! \min _{r>0} \sup _{\widetilde{x} \in \widetilde{M}}\left[\frac{A\left(S_{\widetilde{x}}(r)\right)}{\operatorname{vol}_{B H}\left(B_{\widetilde{x}}(r)\right)}\right]^{n} \operatorname{vol}_{B H}(M) .
$$

By Lemma 8.1 and the volume comparison theorem (Theorem 5.3) we can easily obtain:

Theorem 8.2. Let $(M, F)$ be an $n$-dimensional closed oriented reversible Finsler manifold endowed with the Busemann-Hausdorff volume form. Suppose that weighted Ricci curvature satisfies $\mathbf{R i c}_{N} \geqslant-(N-1)$ for some $N>n$. Then for any $z \in H_{k}(M)$ one has

In particular,

$$
\|z\| \leqslant k !(N-1)^{k} \operatorname{vol}_{B H}(z) .
$$

$$
\|M\| \leqslant n !(N-1)^{n} \operatorname{vol}_{B H}(M) .
$$

Proof. By Theorem 5.3 we see that the function

$$
\frac{\operatorname{vol}_{B H}\left(B_{\widetilde{x}}(r)\right)}{\int_{0}^{r} \sinh ^{N-1} t d t}
$$

is non-increasing. Noticing that $A\left(S_{\widetilde{x}}(r)\right)=\frac{d}{d r} \operatorname{vol}_{B H}\left(B_{\widetilde{x}}(r)\right)$, we have

$$
\frac{A\left(S_{\widetilde{x}}(r)\right)}{\operatorname{vol}_{B H}\left(B_{\widetilde{x}}(r)\right)} \leqslant \frac{\sinh ^{N-1} r}{\int_{0}^{r} \sinh ^{N-1} t d t} .
$$

Since

$$
\lim _{r \rightarrow \infty} \frac{\sinh ^{N-1} r}{\int_{0}^{r} \sinh ^{N-1} t d t}=N-1
$$

the conclusion follows easily by Lemma 8.1 .

Acknowledgements The main part of this work was completed while I was visiting IUPUI during July 2014. I would like to thank Professor Zhongmin Shen for his valuable suggestions and hospitality. The research was supported by the Natural Science Foundation of China (No. 11171139). 


\section{References}

[1] M. Anderson, On the topology of complete manifolds of nonnegative Ricci curvature, Topology 29 (1990), no. 1, 41-55.

[2] D. Bao, S. S. Chern, and Z. Shen, An introduction to Riemann-Finsler Geometry, GTM 200, Springer-Verlag, 2000.

[3] E. Calabi, On manifolds with nonnegative Ricci curvature II, Notices Amer. Math. Soc. 22 (1975), A-205, Abstract No. 720-53-6.

[4] I. Chavel, Riemannian Geometry: A Modern Introduction, Camb. Univ. Press, 1993.

[5] Q. Ding, A new Laplacian comparison theorem and the estimate of eigenvalues, Chin. Ann. Math. Ser. 15 (1994), no. 1, 35-42.

[6] M. Gromov, Volume and bounded cohomology, IHES Publ. Math. 56 (1983), 213-307.

[7] H. P. McKean, An upper bound for the spectrum of $\triangle$ on a manifold of negative curvature, J. Differential Geom. 4 (1970), 359-366.

[8] J. Milnor, A note on curvature and fundamental group, J. Differential Geometry 2 (1968), 1-7.

[9] S. Ohta, Finsler interpolation inequalities, Calc. Var. Partial Differential Equations 36 (2009), no. 2, 211-249.

[10] S. Ohta and K. T. Sturm, Heat flow on Finsler manifolds, Comm. Pure Appl. Math. 62 (2009), no. 10, 1386-1433.

[11] H. B. Rademacher, A sphere theorem for non-reversible Finsler metrics, Math. Ann. $\mathbf{3 2 8}$ (2004), no. 3, 373-387.

[12] Z. Shen, Volume comparison and its applications in Riemann-Finsler geometry, Adv. Math. 128 (1997), no. 2, 306-328.

[13] Lectures on Finsler Geometry, World Sci., Singapore, 2001.

[14] Y. B. Shen and W. Zhao, On fundamental groups of Finsler manifolds, Sci. China Math. 54 (2011), no. 9, 1951-1964.

[15] B. Y. Wu, Volume form and its applications in Finsler geometry, Publ. Math. Debrecen 78 (2011), no. 3-4, 723-741.

[16] Some results on curvature and topology of Finsler manifolds, Ann. Polon. Math. 107 (2013), ni. 3, 309-320.

[17] B. Y. Wu and Y. L. Xin, Comparison theorems in Finsler geometry and their applications, Math. Ann. 337 (2007), no. 1, 177-196.

[18] S. T. Yau, Some function-theoretic properties of complete Riemannian manifold and their applications to geometry, Indiana Univ. Math. J. 25 (1976), no. 7, 659-670.

[19] S. T. Yin, Q. He, and D. X. Zheng, Some comparison theorems and their applications in Finsler geometry, J. Inequal. Appl. 2014 (2014), 107.

Department of Mathematics

MINJIANG UNIVERSITY

FuzHOU 350121

FuJiAn, P. R. ChinA

E-mail address: wubingye@mju.edu.cn; 569405943@qq.com 